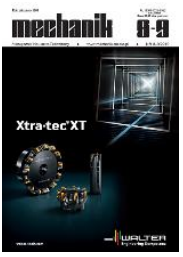

How to cite this article:

Authors: Mateusz Kaszyński, Stanisław Grzywiński, Dariusz Rodzik

Title of article: "Laboratory stand for echolocation signal generating and processing"

Mechanik, No. 8-9 (2019)

DOI: https://doi.org/10.17814/mechanik.2019.8-9.71

\title{
Laboratory stand for echolocation signal generating and processing
}

\author{
MATEUSZ KASZYŃSKI \\ STANISŁAW GRZYWIŃSKI \\ DARIUSZ RODZIK *
}

Mateusz Kaszyński MSc Eng., mateusz.kaszynski@wat.edu.pl - Military University of Technology, Faculty of Mechatronics and Aerospace, Warsaw, Poland

Stanisław Grzywiński PhD Eng., stanislaw.grzywinski@wat.edu.pl, https://orcid.org/0000-0002-3061-608x - Military University of Technology, Faculty of Mechatronics and Aerospace, Warsaw, Poland

Dariusz Rodzik PhD Eng., dariusz.rodzik@wat.edu.pl, https://orcid.org/0000-0003-1697-8874 - Military University of Technology, Faculty of Mechatronics and Aerospace, Warsaw, Poland

The paper presents issues related to the generation and processing of echolocation signals. The conducted simulation studies on the influence of selected system parameters and signal processing methods on the detection capabilities and the accuracy of the determined echolocation coordinates have been described. Particular attention was paid to the presentation of hardware and software solutions for the laboratory stand developed, involving the generation of echolocation signals reflected from imitated objects.

KEYWORDS: digital signal processing, echolocation

\section{Introduction}

Detection of the signal reflected from the object against the background of interference, taking into account the limitations and hardware conditions of the signal path, and determining the coordinates based on the parameters of the useful signal in the ultrasonic band is a difficult task. Ensuring the ability to detect objects with a small effective reflection surface, while maintaining a significant range of instrumental echolocator and high accuracy of determined coordinates, requires development of an efficient and effective system solution of the transceiver track, as well as effective signal processing methods and algorithms of the coordinate determination system [4].

Ensuring the required signal parameters from a hardware point of view - can be achieved by increasing the signal energy using a higher power transmitter. However, this proves to be problematic, taking into account the safety requirements and system limitations of the transmission path. Increasing the total energy of the transmitted signal by extending duration of the probe pulse assuming no additional manipulation of the probe signal and maintaining the peak value of the generated signal pressure - also allows to increase the signal-to-noise ratio. This operation improves the detection range at the expense of ability to distinguish between two or more objects in space [2]. Therefore, designers developing echolocator system solutions based mainly on amplitude detection often have to compromise, and thus reduce the range of the device in favor of maintaining the required range resolution.

Development of digital circuits, especially DDS (direct digital synthesis) and DAC (digital analog converter) as well as signal processors and digital signal processing methods used in echolocation systems, has ensured high echolocation parameters using complex manipulation of probe signal parameters and useful signal processing. One example is the use of complex frequency and phase modulation of the probe signal as well as matched filtering algorithms [1]. This guarantees an effective increase in the signal-to-noise ratio, and thus an increase in the maximum range while maintaining the required range resolution.

To develop echolocator solutions, a detailed analysis is required - both by computer simulation methods and experimental methods. 
The main purpose of the paper is to present the completed simulator and laboratory stand designed to generate signals reflected from virtual objects, as well as to verify the algorithms for processing the echolocation signals.

\section{Simulator of echolocation system}

Verifying the effectiveness of manipulation of the probe signal parameters, the correctness of processed signal reflected from objects and algorithms used in the process of maximizing the signal-to-noise ratio and subsequent estimation of coordinates requires conducting complex simulation tests, taking into account the kinematics of the object's motion and the adopted parameters of the echolocator. The use of modulation allows the spectrum to be expanded irrespective of its duration, and thus a possible increase in the signal-to-noise ratio, obtained after time compression and dependent on the matching filtration gain [5]. To test the detection efficiency of a useful signal, it is necessary, among others, a quantitative assessment of the intensity of the signal reflected from the target. Therefore, in the Matlab environment, a simulator was developed to simulate signals reflected from virtual objects and to test signal processing methods.

The simulation software allows to verify the detection capabilities and determine the coordinates of the echolocator taking into account: parameters of the probe signal modulation, standard noise deviation $\sigma_{\mathrm{x}}$, sampling frequency $f_{\text {pr }}$, time window duration $\tau^{\prime}$, in which $N$ signal samples are taken at once for convolution operations $\left(N=\tau^{\prime} \cdot f_{\mathrm{pr}}\right)$, the time delay of the time window relative to the processed signal is, determined for discrete signals as the amount of shift of the time window relative to the downloaded signal $k$.

Simulation tests were carried out, taking in turn samples of the useful signal occurring against the background of noise contained in the $i$-element table, to the memory buffer of size $N$. Then the samples were subjected to the convolution operation with the response of the matched filter, according to the relationship:

$$
y(n)=x(n) \cdot s(n)=\sum_{n=0}^{N} x(m) s(n-m)
$$

As a result of this operation, from the obtained table of size $(2 N-1)$ containing the resulting values of the signal convolution from the $m$-th download, the value of $y_{\max }(m)$ was determined and recorded into a table with the dimension $j=i / k$. The effect of time window delay with the assumed number of signal samples $k$ was examined. Sample simulation results are shown in fig. 1.

a)

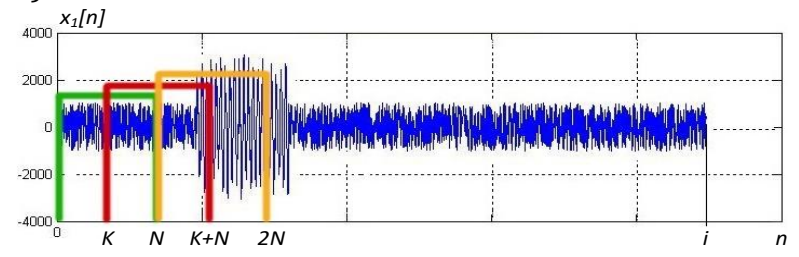

b)

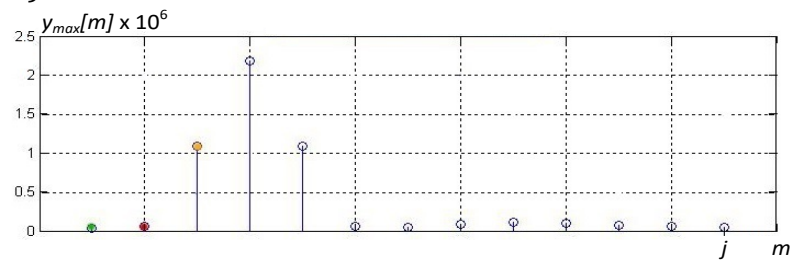

Fig. 1. Method of sampling and sample subjecting to convolution with the response of a matched filter: a) imaging of time windows, b) result of matched filtration

a) Echo signal $\mathrm{x} 1[\mathrm{n}]=\mathrm{s} 1[\mathrm{n}]+\mathrm{n}[\mathrm{n}]$ (narrow band + noise)

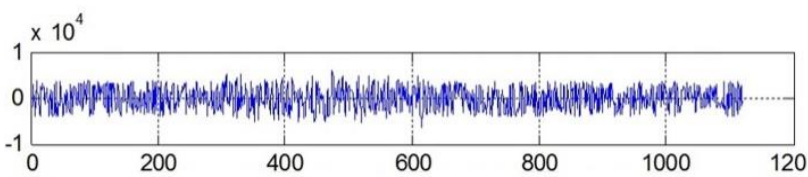

b)

Correlation receiver output ymax1[m] for $\mathrm{x} 1[\mathrm{n}]$

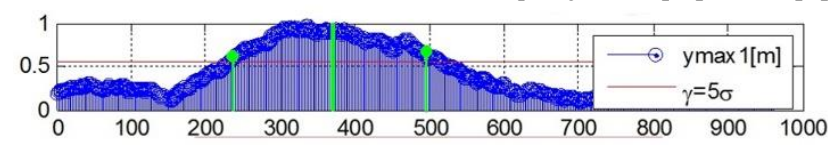

c)

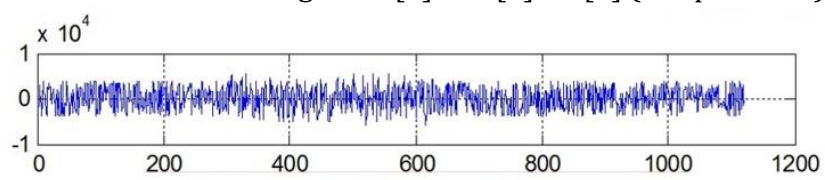

d)

Correlation receiver output ymax $2[\mathrm{~m}]$ for $\mathrm{x} 2[\mathrm{n}]$

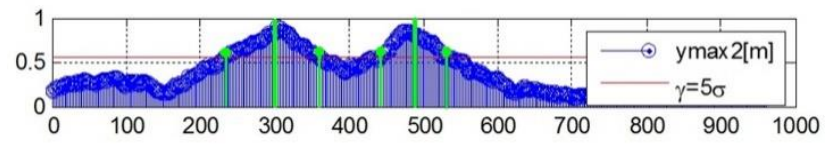

Fig. 2. Comparison of detection capabilities of echo signal processing: a) -b) narrowband, c) -d) with linear chirp signal 
The simulation software was used to analyze the possibilities of increasing the range resolution - tests of the influence of the probe signal parameters and changes of the time window shift with matched filtration were carried out. Sample results of increasing the ability to distinguish objects using signal modulation and digital processing algorithms are presented in fig. 2 .

\section{Laboratory stand}

Simulation of the real signal reflected from the object requires the use of, among others, signal generators arranged in the form of an extended stand. An echolocation imitator of the object was designed and made, allowing the generation of a signal with parameters that depend on the kinematics of the object and parameters of the transceiver path. The stand provides signals reflected from objects, the parameters of which are determined using a developed simulator. This unit reflects the work of the echolocation system by imitating objects, taking into account the conditions of propagation of the acoustic wave in the air. STM32F4 processor capabilities were used, which performs the tasks of communication with simulation software and imitation of objects by generating the echo signals.

The effect of attenuation of the acoustic wave in the air and the simulation of the reflected signal intensity was implemented by changing the amplitude of the echo signal using amplifiers with $40 \mathrm{~dB}$ gain control. Two independent signal paths were used to imitate two objects to be able to conduct research related to range resolution for given parameters of the probe signal and selected processing algorithms. Generated echo signal is processed by an echolocator equipped with matching systems and the same processor with implemented signal processing algorithms (fig. 3).

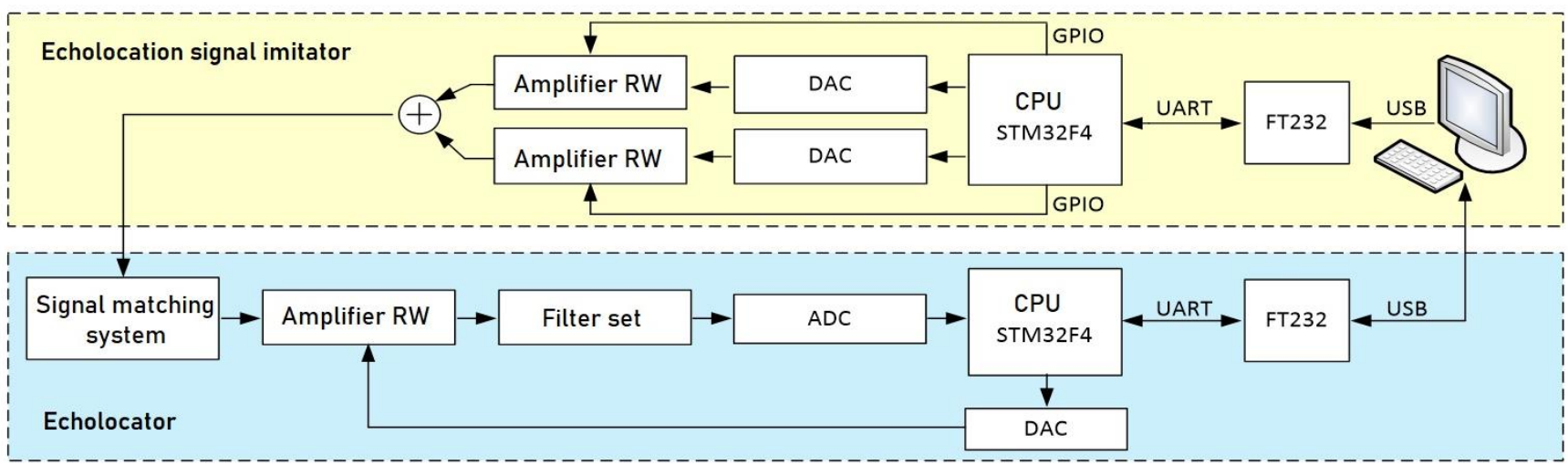

Fig. 3. Functional diagram of the laboratory stand
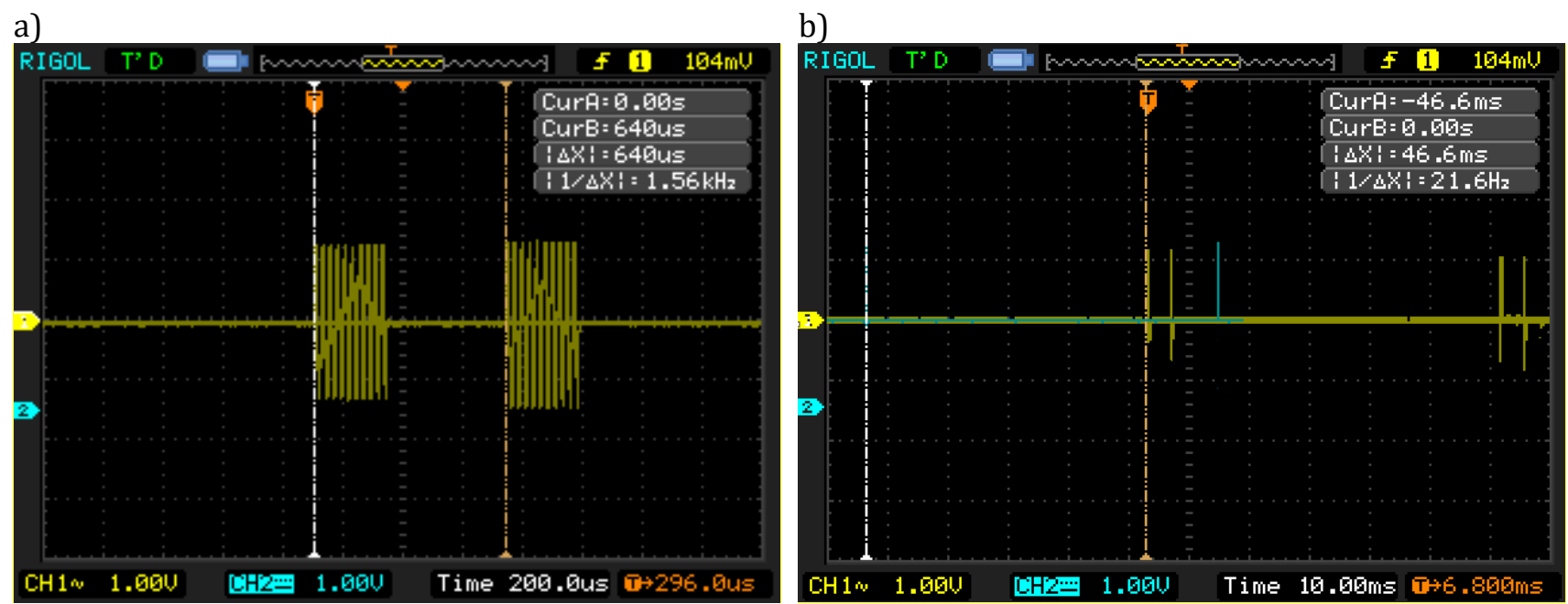

Fig. 4. Oscillograms showing: a) probe signal with echo signal, b) synchronization signals

Examples of oscillograms for the probe signal and the echo signal are shown in fig. 4a, and in fig. 4b - oscillograms of pulses synchronizing the echolocator with the laboratory stand. 
The software implementation of the STM32F4 processor was implemented in the Keil environment with the $\mu$ Vision package. The initiation of any peripheral system using the SPL (standard peripherals library) boils down to:

- calling a function that sets a value in the processor's register, on which the delay of the clock signal to a given peripheral system depends, in order to enable it;

- introducing settings regarding the operating mode and parameters of the initialized peripheral system by assigning register values to appropriate variables belonging to the object structure;

- calling the initialization function, in which selected settings are entered into the processor's registers.

In order to ensure the synchronous operation of the stand and developed echolocator, counter-time systems were used, which indirectly generate synchronization pulses with a specific repetition frequency fi (fig. 4b). Sending a data frame from a PC, containing information about the parameters of imitated objects selected by the user, starts the imitation of the echolocation signals. The proper synchronization and sequence of actions carried out by the processor's peripheral circuits was ensured using the interrupt handling system, in which flags setting subsequent processes were set.

The developed echolocation software uses DMA (direct memory access) systems to directly transfer data from the ADC converter to RAM (random access memory) [3]. In this way, simultaneous processing and matched filtering of the signal from the current time base and the transfer of designated data on detected objects from previous probing were enabled. With this approach, the processes of acquisition, processing, filtration and data transmission can take place in real time.

\section{Summary}

The developed laboratory stand for processing echolocation signals is designed to generate signals reflected from virtual objects with given parameters. The input parameters of the developed imitator are entered by the user and can be changed during the system operation. The imitator allows to enter:

- carrier frequency,

- echolocator repetition frequency,

- pulse duration,

- parameters of imitated objects, i.e. initial distance and speed.

Based on the entered parameters, the parameters of the probing and reflected signals from the virtual object are determined.

The laboratory stand presented in this paper along with dedicated software allows to study the impact of manipulation of probe signal parameters and processing algorithms on the detection efficiency and accuracy of determined coordinates of detected objects.

The stand allows for testing the algorithms and generating signals with non-linear frequency modulation or with discrete phase modulation using algebraic codes (including Barker and Golay). It imitates signals reflected from objects with artificial amplitude suppression assumed, reflecting the manner of wave propagation and reflecting properties of objects. The developed signal processing algorithms are universal and can also be used in other echolocation systems, taking into account the nature of the propagation of waves used and available transceiver design solutions.

\section{REFERENCES}

[1] Żygadło S., Grzywiński S., Achtenberg K. „Control and measuring generator for inspection of selected radar parameters" (in polish). Mechanik. 89, 7 (2016): 880-881.

[2] Salamon R. „Systemy hydrolokacyjne”. Gdańsk: Gdańskie Towarzystwo Naukowe, 2006.

[3] Grzesiak A. „Teoretyczne Podstawy Radiolokacji. Część 1”. Warszawa: WAT, 1981.

[4] Grzywiński S., Achtenberg K. „Using of the FPGA System for DSP Algorithms Implementation” (in polish). Problems of mechatronics. Armament, aviation, safety engineering. 8, 3 (2017): 157-164.

[5] STM32F407VG Datasheet, www.st.com/content/ccc/resource/technical/document/datash/eet/ef/ 92/76/6d/bb/c2/4f/f7/DM00037051.pdf/files/DM00037051.pdf/jcr:content/translations/en.DM00037051.pdf_(19.12.2017). 